\title{
Evaluation of the Potential Effects of Retinol and Alginate/Gelatin-Based Scaffolds on Differentiation Capacity of Mouse Mesenchymal Stem Cells (MSCs) into Retinal Cells
}

\author{
Mahtab Haghighat ${ }^{1}$, Alireza Iranbakhsh ${ }^{1}$, Javad Baharara ${ }^{2}$, \\ Mostafa Ebadi ${ }^{3}$, Fattah Sotoodehnejadnematalahia ${ }^{1}$ \\ ${ }^{1}$ Department of Biology, Science and Research Branch, Islamic Azad University, Tehran, Iran \\ ${ }^{2}$ Department of Biology, Applied Biology Research Center, Mashhad Branch, Islamic Azad University, Mashhad, Iran \\ ${ }^{3}$ Department of Biology, Damghan Branch, Islamic Azad University, Damghan, Iran
}

Background and Objectives: Retinal stem cells (RSCs) resided in ciliary epithelium have shown to possess a high capacity to self-renew and differentiate into retinal cells. RSCs could be induced to differentiate when they are exposed to stimuli like natural compounds and suitable contexts such as biomaterials. The aim of this study was to examine the effects of Retinol and alginate/gelatin-based scaffolds on differentiation potential of mesenchymal stem cells (MSCs) originated from mouse ciliary epithelium.

Methods and Results: MSCs were extracted from mouse ciliary epithelium, and their identity was verified by detecting specific surface antigens. To provide a three-dimensional in vitro culture system, $2 \%$ alginate, $0.5 \%$ gelatin and the mixed alginate-gelatin hydrogels were fabricated and checked by SEM. Retinol treatment was performed on MSCs expanded on alginate/gelatin hydrogels and the survival rate and the ability of MSCs to differentiate were examined through measuring expression alterations of retina-specific genes by ICC and qPCR. The cell population isolated from ciliary epithelium contained more than $93.4 \%$ cells positive for MSC-specific marker CD105. Alginate/gelatin scaffolds showed to provide an acceptable viability (over 70\%) for MSC cultures. Retinol treatment could induce a high expression of rhodopsin protein in MSCs expanded in alginate and alginate-gelatin mixtures. An elevated presentation of Nestin, RPE65 and Rhodopsin genes was detected in retinol-treated cultures expanded on alginate and alginate-gelatin scaffolds.

Conclusions: The results presented here elucidate that retinol treatment of MSCs grown on alginate scaffolds would promote the mouse ciliary epithelium-derived MSCs to differentiate towards retinal neurons.

Keywords: Mesenchymal stem cell, Retinal neurons, Cell differentiation, Alginate, Gelatin, Scaffolds

Received: March 31, 2021, Revised: September 7, 2021,

Accepted: September 8, 2021, Published online: October 31, 2021

Correspondence to Alireza Iranbakhsh

Department of Biology, Science and Research Branch, Islamic

Azad University, Tehran 1477893855, Iran

Tel: +98-21-44865179-82, Fax: +98-21-44865154

E-mail: iranbakhsh@iau.ac.ir

(a) This is an open-access article distributed under the terms of the Creative Commons Attribution Non-Commercial License (http://creativecommons.org/ licenses/by-nc/4.0/), which permits unrestricted non-commercial use, distribution, and reproduction in any medium, provided the original work is properly cited.

Copyright (c) 2022 by the Korean Society for Stem Cell Research

\section{Introduction}

Retinal stem cells (RSCs) and progenitor cells (RPCs) are the adult multipotent cells which are capable of self-renewal and differentiation into almost all retinal cells (1). They were firstly discovered in lower vertebrates like urodele amphibians (2) and teleost fishes (3), which are located at a region called the ciliary marginal zone (CMZ) between neural retina and ciliary epithelium and present a high potential to proliferate and to generate retinal cells throughout life (4). Interestingly, it has been recognized 
that the stem cells resided in CMZ of frogs and fishes could be able to completely restore the retina after severe damages or retinal detachments (5). The previous studies showed that the chick embryos also possess the CMZ containing multipotent stem cells with a potent capacity to renew the damaged retina, however, it was found that the regenerative potential of these retinal stem cells has been drastically reduced in postnatal and adult chickens (6). Although the mammalian retina lacks a regenerative source of stem cells like CMZ, it was identified that there are more restricted stem cells and progenitors in ciliary margin of the peripheral retina, which could proliferate and differentiate into retinal neurons under proper conditions (7). It was previously reported that the adult stem cells are able to be isolated from the ciliary body epithelium of mouse (8) and human eye (9) and proliferate in in vitro conditions. Moreover, their progenies showed a multipotent capable of reprogramming and generating mature retinal cells such as photoreceptors, ganglion cells, bipolar cells, amacrine cells and retinal pigmented epithelium (RPE) cells $(8,10,11)$. Therefore, it seems that RSCs and RPCs could be considered as promising candidates for stem cell therapies for retinal degenerations. As previous studies showed that RSCs transplanted into retina might differentiate into undesired cells or not integrate in neuronal layers of retina, it was suggested that in vitro induction of RSC differentiation into progenitor cells or precursors of retinal neurons like photoreceptors may be necessary to achieve fully differentiated photoreceptors and retinal integration $(12,13)$. In this regard, many studies have been conducted to differentiate RSCs towards retinal neurons through gene modulation (14) or treatments with various effective substances such as taurine, retinoid acid and carotenoids (15), and different growth factors like FGF-2 (16). Natural compounds like plant secondary metabolites have shown to have effects on proliferation, cell signaling pathways, apoptosis and differentiation processes (17). Retinol also known as vitamin Al is a natural supplement that used to treat vitamin A deficiency and exhibits crucial functions in regulation of several cellular processes including proliferation, apoptosis, differentiation and development. Previous studies have reported that all active forms of retinoids including retinol act as signaling molecules that affect nearly every cell type, demonstrate profound effects on development and mediate cell differentiation by binding to RAR/RXR proteins and activating their signaling pathway. In cancer therapies, retinoids are also used in cancer chemotherapeutic regimens. It has been revealed that retinoids and their other active forms could induce tumor cell differentiation and activate proteins that sensitize tumor cells to anti-cancer drug combinations (18). Therefore, finding out the mechanisms underlying retinoid functions in cell signaling pathways would improve therapeutic strategies for many diseases such as cancer and inflammatory diseases. Apart from impressive natural or synthetic compounds, development of different biocompatible cell scaffolds as 3D culture systems is addressed as a subject of intense investigations in both in vitro and in vivo cell therapies (19). Currently, a plethora of biomaterials applicable as cell scaffolds including natural or synthetic polymers, ceramics, and metal-composite scaffolds have been developed and employed in tissue engineering studies (20). Among these scaffolds, alginate/gelatin-based hydrogels are one of the most suitable scaffolds that provide a 3D environment resembled that of many tissues (21). Alginate is a natural polysaccharide obtained from brown algae, and gelatin is a natural polymer derived from collagen, which are widely utilized in tissue engineering applications due to their proper features including biodegradability, biocompatibility, aqueous solution solubility, hydrophilic properties $(22,23)$.

In current study, we evaluated the effects of Retinol added to differentiation medium and alginate/gelatinbased scaffolds on differentiation ability of mouse ciliary epithelium-derived MSCs into retinal cells.

\section{Materials and Methods}

\section{Isolation and expansion of MSCs from the mouse ciliary body epithelium}

The Balb C mice (6 8 weeks old, 23 25 g weight) were purchased from Pasteur Institute of Iran. The care and use of animals were followed according to the standard protocols provided for working with laboratory animals. All animals were housed in controlled rooms providing a normal temperature $\left(22^{\circ} \mathrm{C}\right)$, humidity $(45 \%)$, 12-h light cycles, and enough food and water. The animals were treated according to the instructions determined by Ethical Committee of Islamic Azad University for working with laboratory animals.

To extract the desired cells from mouse eyes, the mice were firstly euthanized with ketamine/xylazine and then the eye globes were enucleated and placed into a sterile plate containing Artificial Cerebral Spinal Fluid (ACSF). Under a stereo microscope, surrounding tissues including connective and adipose tissues were separated and the clean eye globes immersed in ACSF were subjected to a vertical excision with a dissecting scissor. The two obtained hemispheres were naked from internal parts of eye 
including vitreous fluid, lens, and neuroretina. After removal of the cornea and iris, the ciliary body section was then carefully separated and incubated at first in $2 \mathrm{ml}$ of $0.5 \%$ Dispase (Sigma, Germany) solution at $37^{\circ} \mathrm{C}$ for 10 min and then in trypsin, hyaloronidase and kinortic acid (Sigma, Germany). The mixture of ciliary body epithelium and enzymes was poured into a $14 \mathrm{ml}$ tube and ciliary epithelium was dissected to single cells through pipetting. To separate the single cells, centrifugation was done at 1,500 rpm for $5 \mathrm{~min}$ and the cell pellet was suspended into a serum-free culture medium containing trypsin inhibitor. The second centrifugation was done at $1,500 \mathrm{rpm}$ for 5 min and the cells were suspended in $1 \mathrm{ml}$ of culture medium. Ultimately, a density of 10 cells per $1 \mu 1$ were seeded in a 24 -well plate containing $500 \mu 1$ of DMEM12 medium (GE Healthcare Life Sciences, USA) $+10 \%$ FBS and incubated at $37^{\circ} \mathrm{C}$ for one week.

\section{Characterization of cell population isolated from ciliary body epithelium of mouse eyes}

The cells isolated from ciliary body epithelium were defined by detection of specific surface antigens like CD105 and CD45. For this aim, the expanded cells (passage 4) were detached from culture dish using $0.25 \%$ trypsin/ EDTA solution (Sigma, Germany), centrifuged at 1,500 rpm for $5 \mathrm{~min}$ and the pellet was resuspended in $1 \%$ bovine serum albumin (BSA) in PBS. Next, the cells were incubated with primary antibodies (Abcam; UK, $1: 100$ ratio) at $4^{\circ} \mathrm{C}$ for 1 hour and then centrifuged at $1,500 \mathrm{rpm}$ for $5 \mathrm{~min}$. Afterwards, the cell suspension were exposed to the secondary antibody fluorescein isothiocyanate (FITC) conjugated anti-mouse (Abcam; UK, $1: 200$ ratio) for $45 \mathrm{~min}$ at room temperature in the dark. The cells were lastly rinsed with PBS and evaluated using a flow cytometer.

\section{Design and fabrication of alginate/gelatin-based scaffolds}

Three types of scaffolds including gelatin, alginate and alginate/gelatin (Alg-Gel) hydrogels were fabricated according to following procedures.

\section{Alginate gel}

To find the most proper concentration, the alginate hydrogels were constructed at three concentrations of $0.5 \%$, $1 \%$ and $2 \%$. To do this, sodium alginate (alginate 1) and sodium alginic acid salt from brown algae (alginate 2) (Sigma-Aldrich, Germany) were dissolved in $150 \mathrm{ml}$ of PBS ( $\mathrm{pH}$ 7.0). The solutions were stirred at room temperature overnight. For hybrid alginate gels, hyaloronate so- dium (NaHa) or polyethylene glycol (PEG) and their associated compounds were used (Sigma-Aldrich, Germany). After dissolving alginate, PEG or $\mathrm{NaHa}$ was added and blended overnight to gain a homogeneous fluid. The hydrogels were prepared at unsterile conditions to evaluate the composition and mechanical stability of hydrogels. For cell study, all prepared solutions were filtered using $0.2 \mu \mathrm{m}$ filters.

\section{Gelatin gel}

To find the suitable concentration, the gelatin hydrogels were constructed at three concentrations of $0.25 \%, 0.5 \%$ and $1 \%$ by dissolving $50 \mathrm{mg}, 100 \mathrm{mg}$ and $200 \mathrm{mg}$ gelatin polymer (Sigma-Aldrich, Germany) into $20 \mathrm{ml}$ of $2 \%$ acetic acid (v/v), respectively. The solutions were then mixed by a hot-plate magnetic-stirrer until absolutely dissolved. To solidify the fabricated polymers, the solutions were transferred into two mirror tubes and gathered at $-18^{\circ} \mathrm{C}$ overnight. Afterwards, to remove the solvent and achieve porous scaffolds, the solutions were incubated in a lyophilizer for 48 hours (LABCONCO-Freeze System, Kansas city, MO, USA). The fabricated scaffolds were ready after breaking the glass tubes and consequently branched into two parts for next evaluations.

\section{Alginate/gelatin (Alg-Gel) gel}

According to the results obtained from cytotoxicity assays, the most ideal concentration of each alginate and gelatin hydrogel was elected to fabricate the blended alginate/gelatin (Alg-Gel) hydrogel. To do this, a Vaseline bath with vacant gapes was provided and cooled at $-18^{\circ} \mathrm{C}$. Next, 10\% Na-Alg solution (Sigma-Aldrich, Germany) were transferred into holes and stored at $-18^{\circ} \mathrm{C}$ for 15 min. Na-Alg solid particles were subjected to cross-linking reaction in four concentrations of $\mathrm{CaCl} 2$ solution (Merck) for one hour. Afterwards, the beads were mixed up, washed with the distilled water and then dried at the air and also into a vacuum oven at 30 . Regarding to different concentrations of $\mathrm{CaCl} 2$ solution, a mean diameter around $0.8 \pm 0.2 \mathrm{~cm}$ was obtained. Na-Alg hydrogel beads were covered by gelatin through submerging in gelatin solution. The gelatin-coated beads were then dried at $30^{\circ} \mathrm{C}$ and kept for latter assays.

\section{Alginate/gelatin scaffold characterization}

The structure, morphology and porosity distribution of alginate, gelatin and alginate/gelatin hydrogels were evaluated by scanning electronic microscope (SEM). To prepare the samples, the surfaces of hydrogels were covered with a thin layer of gold and inserted into the SEM 
chamber. The prepared samples were visualized at a certain magnitude.

\section{Culture and visualization of MSCs on alginate/gelatin-based scaffolds}

The MSCs at a density of $1 \times 10^{5}$ cells per well were cultured on alginate, gelatin and alginate/gelatin (Alg-Gel) scaffolds providing $3 \mathrm{D}$ culture systems and also grown as a monolayer in a flat dish (2D culture). To detect MSCs on scaffolds, the cell nuclei were stained with DAPI (4', 6-diamidino-2-phenylindole, Thermofisher, USA). Briefly, the cells at a density of $1 \times 10^{4}$ cells per well were implanted on alginate, gelatin and alginate/gelatin (Alg-Gel) scaffolds. On next day, the culture medium was discarded, the cells were rinsed by PBS and then incubated with DAPI for $20 \mathrm{~min}$ in the dark. After washing once in PBS, the cells were examined under a fluorescent microscope (Nikon, Japan).

\section{Retinol treatment}

To examine the differentiation potential of MSCs, a differentiation medium consisted of $50 \mu \mathrm{M} \beta$-mercaptoethanol, $1 \times$ minimum essential medium-nonessential amino acids (Thermo Fisher Scientific) and 20\% of knockout serum substitution (KSR, Thermo Fisher Scientific) was applied as a basic differentiation medium to allow cells grow and differentiate in 3D and 2D culture systems. Moreover, the differentiation medium supplemented with Retinol was used to evaluate the effects of Retinol on MSC differentiation. For this aim, the cell at a density of $1 \times 10^{4}$ cells per well were cultured in a 24-well plates as four groups including (1) Retinol-treated cells cultured on alginate/gelatin (Alg-Gel) scaffolds (Retinol + alginate/gelatin), (2) Retinol-treated cells cultured as monolayer (Retinol+ 2D), (3) Differentiation medium-treated cells cultured on alginate/gelatin (Alg-Gel) scaffolds (Dif + alginate/gelatin), and (4) Differentiation medium-treated cells cultured as monolayer $(\mathrm{Dif}+2 \mathrm{D})$. The $3 \mathrm{D}$ and $2 \mathrm{D}$ cultures treated with the differentiation medium alone were considered as control groups and the treatment groups were treated with differentiation medium supplemented by $5 \mu \mathrm{M}$ Retinol (Sigma-Aldrich, Germany) and incubated at $37^{\circ} \mathrm{C}(5 \%$ CO2) for 21 days.

\section{MTT assay}

Three different concentrations of alginate $(0.5 \%, 1 \%$, and $2 \%)$ and gelatin $(0.25 \%, 0.5 \%$, and $1 \%)$ were prepared and the viability of cells seeded on them were measured by MTT. Briefly, the fabricated scaffolds were put in a 96-well plate and $1 \times 10^{4}$ cells per well were cultured on each scaffold. For 2D culture, the cells were directly cultured in the floor of wells. For MTT assay, the medium was removed, $200 \mu 1$ of MTT solution $(0.5 \mathrm{mg} / \mathrm{ml})(\mathrm{MP}$ Biomedicals, LLC, Santa Ana, CA, USA) was eked to wells and cells were incubated at $37^{\circ} \mathrm{C}$ for 4 hours in the dark. Then, the medium was removed and $200 \mu 1$ of DMSO (MP Biomedicals, LLC) was poured to solve formazan crystals. Eventually, the color absorbance was meted at $492 \mathrm{~nm}$ by an Eliza reader (EX800, USA). Moreover, the effect of the differentiation medium alone (control group) and differentiation medium perfected with $5 \mu \mathrm{M}$ Retinol on the viability of MSCs grown on the selected densities of alginate, gelatin and alginate-gelatin hydrogels was evaluated by MTT as described above.

\section{Functionality assay}

The in vivo functionality of the putative differentiated cells was also assessed as described below. A total of 12 adult male Wistar rats weighing 280 300 g were used. The animals were housed under the standard condition $\left(22 \sim 24^{\circ} \mathrm{C}, 45 \sim 50 \%\right.$ humidity, $12 \mathrm{~h}: 12 \mathrm{~h} \mathrm{light/dark}$ cycle) with free access to food and water. During the in vivo study, the animals were behaved according to ethical instructions of working with laboratory animals defined by the research ethics committee of Azad University. The rats were dark-adapted overnight, pupils were dilated with cyclogyl 1\% (Alcon Pharmaceuticals, Fribourg, Iran) and phenylephrine 5\% (Ursapharm, Saarbrücken, Germany) in dim red light. Approximately $5 \mathrm{~min}$ before exposure, the rat were anesthetized subcutaneously with ketamine (85 mg/kg; Inresa Arzneimittel, Freiburg, Germany) and xylazine (4 mg/kg Bayer AG, Leverkusen, Germany) and located on a pre-warmed surface. Keeping both eyes moist during exposure, $2 \%$ methocel (OmniVision AG, Neuhausen, Switzerland) was applied. The left eyes were exposed to blue light $\left(410 \pm 10 \mathrm{~nm} ; 60 \mathrm{~mW} / \mathrm{cm}^{2}\right.$ at the level of the cornea) for 10 30 min. The right (unexposed) eyes were served as controls. Following light exposure, the rats were kept in darkness overnight and then returned back to the normal light/dark cycle until analysis (24). The rats were divided into 4 groups ( $n=3$ per group) as follows: (1) The control group (Diff+2D) in which rats received the cells 2D-cultured in differentiation medium with no retinol, (2) The Diff + Alginate/Gelatin group in which rats received those cells cultured on Alginate/Gelatin scaffold (3D culture system) in differentiation medium with no retinol treatment, (3) The Retinol+2D group in which rats were transplanted by the cells cultured in differentiation medium supplemented with Retinol, and (4) The Retinol+ Alginate/Gelatin group in which rats received cells cul- 
tured on Alginate/Gelatin scaffold (3D culture system) in differentiation medium supplemented with Retinol. The differentiated cells of both 2D and 3D groups were suspended in DMED medium (Sigma, Germany) at a density of $5 \times 10^{4}$ cells/ $\mu 1$ and $10 \mu 1$ were injected into the subretinal space of the left eye $(n=12)$, using a 30-gauge needle attached to a Hamilton syringe. Control (sham) groups received an equivalent volume of DMED medium. The animals were evaluated for visual function 14 days post-transplantation. Transplantation efficacy was assessed by optokinetic testing whereby an animal that could see will involuntarily move its head in response to a moving stimulus.

Furthermore, the location of the transplanted differentiated cells was examined via tracking the expression of rhodopsin protein in the transplanted cells using immunohistochemistry assay (25).

\section{qPCR quantification}

The probable alterations in expression levels of three Nestin, RPE65 and Rhodopsin genes were meted in Retinol-treated MSCs and control group (differentiation medium), which expanded on $2 \%$ alginate, $0.5 \%$ gelatin and alginate/gelatin (Alg-Gel) scaffolds and also as a monolayer. Briefly, total RNAs were extracted from all samples using Tripure isolation reagent instruction (SigmaAldrich, Germany) and were subjected to cDNA synthesis reaction by using cDNA synthesis kit (Thermoscientific, UK). For Real-Time PCR, the primers were designed by Gene runner software (Version 6.5.52) and their efficiency and specificity were analyzed by BLAST tool (Table 1). The amplification reaction was done using 2X Real-Time PCR Master Mix (BioFACT ${ }^{\mathrm{TM}}$, Korea) and an Applied Biosystems StepOne. Beta-actin gene was applied as the housekeeping gene for normalization of the expression levels of the studied genes. The PCR thermal program was set as follows: $95^{\circ} \mathrm{C}$ for $15 \mathrm{~min}$ as initial denaturation, followed by an amplification step for 40 cycles with denaturation segment at $95^{\circ} \mathrm{C}$ for $15 \mathrm{sec}$, annealing segment at $60^{\circ} \mathrm{C}$ for $30 \mathrm{sec}$ and extension segment at $72^{\circ} \mathrm{C}$ for $30 \mathrm{sec}$. The melting curve analysis was also done at the end of the amplification step. All qPCR experiments were performed in triplicate, and the relative gene expressions were calculated through the $2^{-\Delta \Delta \mathrm{Ct}}$ method.

\section{Immunocytochemistry assay}

The neural differentiation of MSCs treated with Retinol, compared to differentiation medium alone, was evaluated by detecting rhodopsin protein as a photoreceptor-specific marker using ICC assay. In brief, $1 \times 10^{5}$ cells per well were cultured on $2 \%$ alginate, $0.5 \%$ gelatin and alginate/gelatin (Alg-Gel) hydrogels and treated with Retinol for 21 days. The $2 \mathrm{D}$ cultures were also provided as controls. The ICC assay was performed through a standard protocol described previously (26). Finally, the cells stained by FITC-conjugated antibody (Abcam, $1: 100$ ratio) and DAPI (Thermofisher, USA) were observed under a fluorescent microscope equipped with DAPI and FITC filters (Olympus, Japan).

\section{Statistical analysis}

All experiments were performed in triplicate and the results were represented as the mean \pm standard deviation. To evaluate the statistical significance of the observed differences between treatment and control groups, ANOVA analyses were exerted using GraphPad Prism (Version 5). $\mathrm{p}<0.05$ was considered as significant.

\section{Results}

\section{The features of MSCs isolated from the ciliary body epithelium of mouse eyes}

The cell population isolated from mouse ciliary body epithelium and seeded as monolayer (2D culture) illustrated the classic morphology of mesenchymal stem cells (MSCs), adherence features and colony-like forming units (shown in Fig. 1A). Furthermore, the characterization of the surface antigens of the isolated cells showed that over $80 \%$ of cell population were affirmative for MSC-specific markers (93.4\% for CD105), implying on MSCs as the dominant population of the isolated cells (shown in Fig. 1B). Nevertheless, a few number of cells were CD45+ $(2.55 \%)$ that are considered as specific marker for hematopoietic stem cells.

Table 1. Sequences of the primers used for real-time PCR

\begin{tabular}{lll}
\hline \multicolumn{1}{c}{ Gene } & \multicolumn{1}{c}{ Forward $\left(5^{\prime}-3^{\prime}\right)$} & \multicolumn{1}{c}{ Reverse $\left(5^{\prime}-3^{\prime}\right)$} \\
\hline Gapdh & AAGTTCAACGGCACAGTCAAGG & CATACTCAGCACCAGCATCACC \\
Nestin & AGGGCTGGGAAGAAGAGGAG & GATTGGGATGGGTGAACAGGGT \\
Re 5 ho & TGTTGCTGGAAAGGGTTTGA & TGGCTGTGGCAGTTGTATGG \\
\hline
\end{tabular}




\section{The characteristics of alginate/gelatin-based scaffolds}

The microstructure and porosity properties of the fabricated $2 \%$ alginate, $0.5 \%$ gelatin and alginate-gelatin (Alg-gel) hydrogels were examined by SEM. The SEM images indicated that $2 \%$ alginate, $0.5 \%$ gelatin and the mixed alginate-gelatin (Alg-Gel) hydrogels consisted of the attached to gather, porous constructions appropriate for 3D cell cultures (Fig. 2A). Furthermore, fluorescent images of DAPI staining showed that MSCs were properly

A

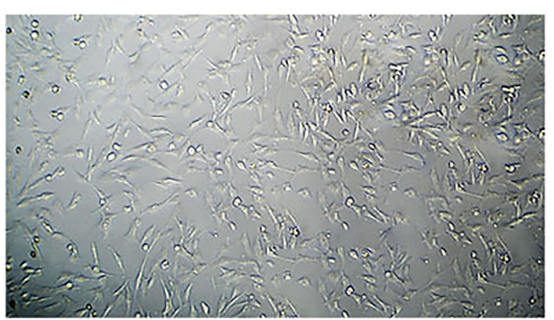

B
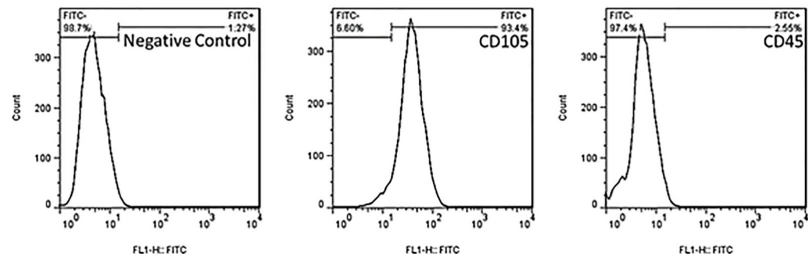

Fig. 1. (A) MSC cultures (passage 4) derived from mouse ciliary body epithelium. The cells grown as monolayer showed the typical morphology and adherence features. Scale bars: $200 \mu \mathrm{m}$. Characterization of the cell population isolated from ciliary epithelium. (B) The dominant population in the isolated cells were positive for MSC-specific marker CD105 (93.4\%) and few cells were $\mathrm{CD} 45+$ (hematopoietic stem cell markers, $2.55 \%$ ).

expanded on the scaffolds of interest (shown in Fig. 2B).

\section{Viability of MSCs on alginate/gelatin-based scaffolds and in differentiation media}

The results from the comparative study of the effects of various densities of alginate and gelatin on viability of MSCs demonstrated that there were no significant differences between alginate $0.5 \%, 1 \%$ and $2 \%$ and that an acceptable cell viability $(>80 \%)$ was provided by all alginate concentrations (shown in Fig. 3). The viability of MSCs cultured on $0.25 \%$ and $0.5 \%$ gelatin was adequately high $(91 \%$ and $83 \%$, respectively) rather than $1 \%$ gelatin $(\sim 65 \%)$. On the Basis of this comparative assay, $2 \%$ alginate and $0.5 \%$ gelatin were chosen for fabrication of the mixture of alginate-gelatin (Alg-gel) scaffold. In next step, the data obtained from MTT assay of two MSC groups treated with differentiation medium alone (control) and supplemented with Retinol, which grown on $2 \%$ alginate and $0.5 \%$ gela-

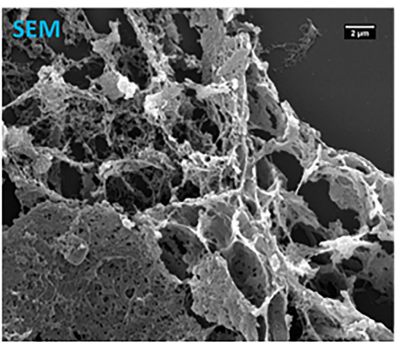

Fig. 2. (A) SEM images of the structural features of alginate/gelatin-based scaffolds. (B) The expansion of MSCs on alginate/gelatin-based scaffolds. The position of MSCs populated on all scaffolds was visualized after DAPI staining the nuclei of MSCs.
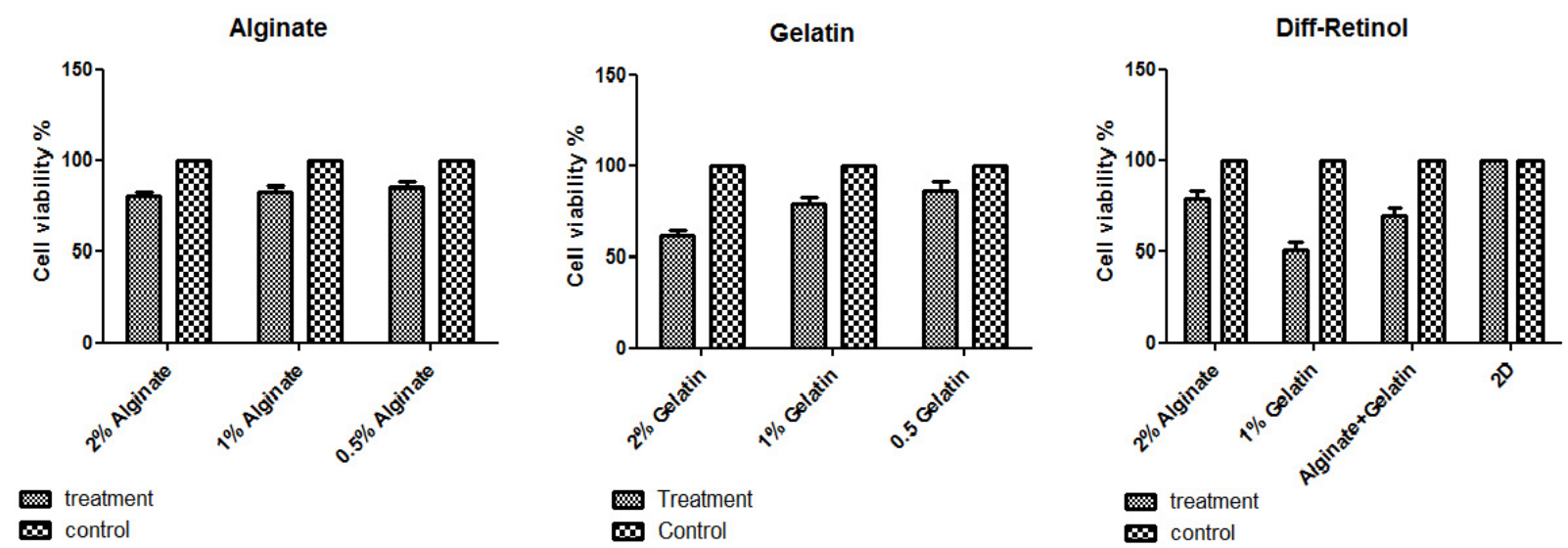

Fig. 3. The viability of MSCs on different concentrations of alginate/gelatin-based scaffolds and 2D cultures. The viability of the MSCs grown on $2 \%$ alginate, and alginate-gelatin scaffolds was significantly higher $(\sim 80 \%)$ than $0.5 \%$ gelatin $(<60 \%)$. Moreover, the $2 \mathrm{D}$ cultures of the Retinol-treated MSCs and control cells (treated with differentiation medium alone) showed a higher viability comparing to 3D culture, suggesting 3D scaffolds could be a suitable context for MSC differentiation. 
tin and alginate-gelatin scaffolds or as a monolayer (2D culture) elucidated that the viability of MSCs broadened on both $2 \%$ alginate and alginate-gelatin mixture scaffolds was significantly higher $(\sim 80 \%)$ than that obtained from $0.5 \%$ gelatin $(<60 \%)$. Furthermore, the data resulted from comparative analyses between $3 \mathrm{D}$ and $2 \mathrm{D}$ culture systems implicated that the Retinol-treated MSCs and control cells (treated with differentiation medium alone) grown as $2 \mathrm{D}$ culture showed a higher viability than those cells expanded on 3D culture, suggesting 3D scaffolds might provide a more ideal context for MSC differentiation.

\section{The expression of retinal cell-specific genes in Retinol-treated MSCs grown on alginate/gelatin scaffolds}

At mRNA level, the relative expression of multiple retina-specific genes including Nestin, RPE65 and Rhodopsin was calculated in Retinol-treated MSCs grown on alginate/gelatin-based hydrogels as 3D culture and 2D culture, comparing to control group (treated with differentiation medium alone). The qPCR data demonstrated that the expression levels of Nestin, RPE65 and Rhodopsin genes were elevated in both treated-cell groups (Retinol and differentiation medium alone) which were expanded on alginate and alginate-gelatin mixture compared to monolayer cultures (2D culture). There was an exception for gelatin that showed no significant difference at the expression level of differentiation-specific genes compared to
2D cultures. Among three kinds of the studies scaffolds, it was observed that expressions of Nestin, RPE65 and $R h o d o p s i n$ genes were significantly upregulated in all treated cultures (Retinol and differentiation medium alone) grown on alginate and alginate-gelatin scaffolds in comparison to gelatin hydrogel. No significant difference in expression level of Nestin, RPE65 and Rhodopsin genes was observed between the MSCs cultured on alginate and alginate-gelatin scaffolds. On the other hand, the real-time PCR data represented a marked upregulation of Nestin, RPE65 and Rhodopsin genes in Retinol-treated MSCs grown on $3 \mathrm{D}$ cultures comparing to control group (differentiation medium), confirming the stimulatory effects of Retinol on in vitro MSC differentiation (shown in Fig. 4).

\section{The expression of retina-specific marker in Retinol-treated MSCs grown on alginate/gelatin-based scaffolds}

In immunochemistry test, the effects of Retinol treatment and differentiation medium alone and alginate/gelatin-based scaffolds as 3D culture and also 2D culture on the expression of rhodopsin protein (a special marker of photoreceptors) were examined in MSC-derived cell cultures. The fluorescent images illustrated that the retinol treatment mainly caused a noticeably higher expression of rhodopsin protein comparing to the differentiation medium alone (shown in Fig. 5A). Furthermore, the quantified ICC data showed that in the Retinol-treated cell groups, those cells grown on alginate and then algi-
RPE65

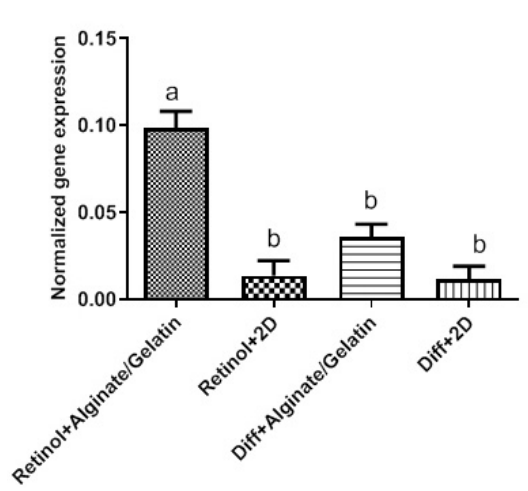

Nestin

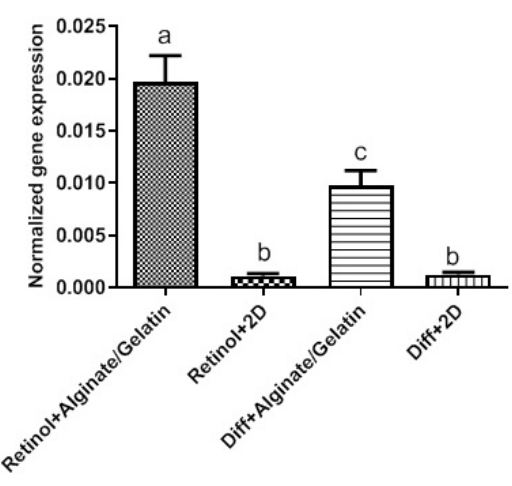

Rhodopsin



Fig. 4. The relative expression of Nestin, RPE65, and Rhodopsin genes in Retinol-treated MSCs cultured on alginate/gelatin-based scaffolds. The expression levels of Nestin, RPE65 and Rhodopsin genes were elevated in both cell groups treated with differentiation medium with and without Retinol, which were grown on alginate and the mixed alginate-gelatin hydrogels compared to monolayer cultures (2D culture). Compared to gelatin, the expression of Rhodopsin and RPE65, Nestin genes was significantly unregulated in all treated cells grown on alginate and alginate-gelatin scaffolds. Importantly, the qPCR data revealed a remarkable elevated expression of Nestin, RPE65 and Rhodopsin genes in Retinol-treated MSCs grown on 3D cultures compared to control group (differentiation medium alone). Statistical analyses were exerted using ANOVA test and $\mathrm{p}<0.05$ was considered significant. 
nate-gelatin scaffolds expressed rhodopsin protein at a significant higher level ( $41 \%$ and $43 \%$, respectively) than those expanded on gelatin and 2D culture (24\% and 18\%, respectively) (shown in Fig. 5B), implying on the effectiveness of retinol treatment and either alginate hydrogel alone or mixed with gelatin on induction of differentiation potential of MSCs.

\section{Visual function of the in vitro differentiated cells in a rat model of retinal degeneration following cell transplantation}

We used the head-tracking response to assess the visual function of the in vitro differentiated cells 14 days after transplantation in left eye of a rat model of retinal degeneration (Fig. 6). Preservation of the higher spatial frequency $(0.5 \mathrm{c} / \mathrm{d})$ monocular optokinetic head-tracking response was associated with the differentiated cells transplanted in subretinal region of Retinol+Alginate/Gelatin $(0.4167 \pm 0.14)$ and Diff + Alginate/Gelatin $(0.2833 \pm 0.02)$ compared to retinal $+2 \mathrm{D}(0.1833 \pm 0.07)$ and Diff $+2 \mathrm{D}$ $(0.0366 \pm 0.05)$ groups. Visual acuity improvement was statistically significant just in Retinol + Alginate/Gelatin group. Conservation of visual acuity in the transplanted eyes was associated with the preservation of photoreceptors in the host outer nuclear layer (ONL). The IHC images showed the incorporation of the differentiated cells in ONL of the transplanted eyes, which were labeled with DiI stain. Furthermore, the expression of rhodopsin protein was detected in the transplanted cells in eyes, which was higher in Retinol+Alginate/Gelatin group compared to other groups (Fig. 7).

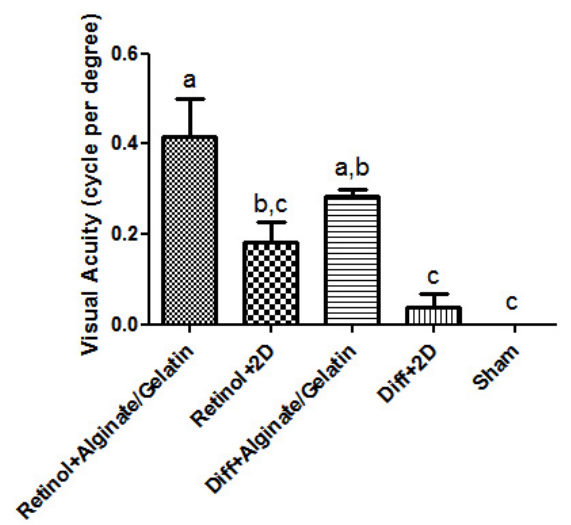

Fig. 6. Preservation of visual function following the differentiated cell transplantation in a rat model of retinal degeneration. Preservation of optokinetic head-tracking response to a rotating vertical stimulus in 8-week-old rats were analyzed following the transplantation of the differentiated cells. Mean visual acuity of the transplanted eye versus control-injected eye and non-transplanted dystrophic eye. Visual Acuity is indicated in cycles per degree (c/d).
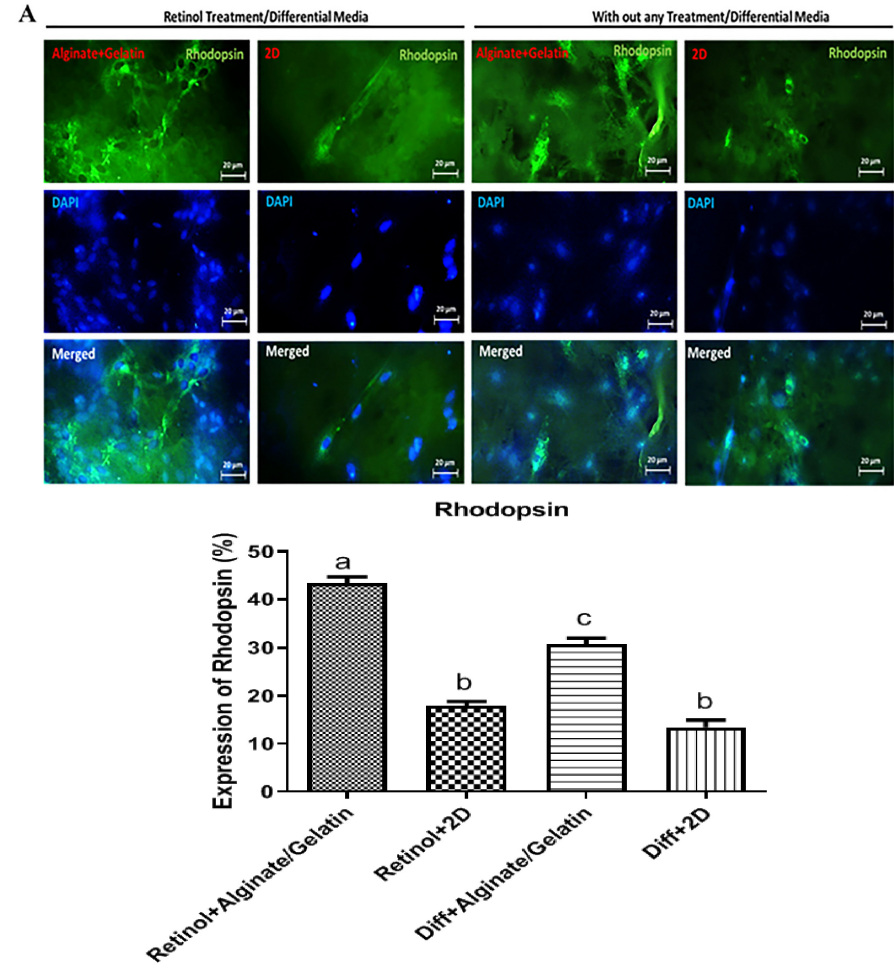

Fig. 5. (A) The cells expressing rhodopsin protein in differentiation medium supplemented with or without Retinol on alginate/gelatin-based hydrogels. Immunofluorescence images showed that the cells treated with Retinol exhibited an increased expression of rhodopsin compared to those treated with the differentiation medium alone. Among three scaffolds, those treated cells grown on alginate and alginate-gelatin scaffolds, respectively, expressed rhodopsin at a significant higher level than those grown on gelatin and 2D culture. FITC, DAPI, and the merged images were represented. Scale bars: $20 \mu \mathrm{m}$. (B) The quantitative data of ICC assay were represented as a statistical graph. Data were statistically analysed by ANOVA test and $p<0.0001$ was considered significant and showed with different letters. 


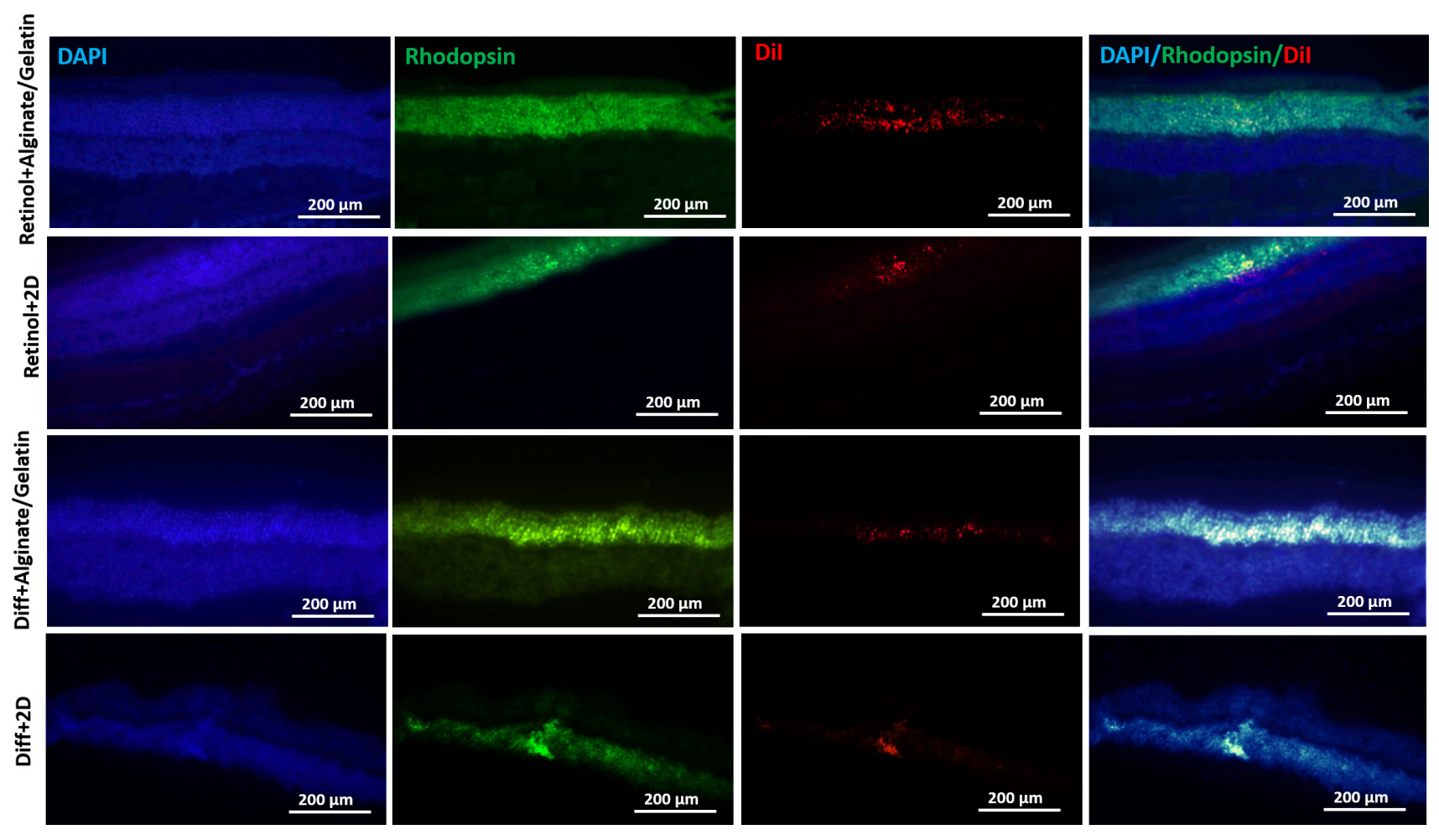

Fig. 7. Immunohistochemically tracking of the transplanted cells and rhodopsin expression. The IHC images showed the incorporation of the differentiated cells in outer nuclear layer of retina in the transplanted eyes (Dil-labeled cells). The expression of rhodopsin protein was also detected in the transplantation region of in vitro differentiated cells, which was higher in Retinol + Alginate/Gelatin group compared to other groups. FITC, DAPI, Dil, and the merged images were represented. Scale bars: $200 \mu \mathrm{m}$.

\section{Discussion}

During last decades, it has been explored that lower vertebrates such as newt and zebrafish manifest an incredible capacity to reconstruct their damaged retina by constantly generating new neurons instead of the lost ones throughout their lives. This great ability was found to be due to the presence of a stem cell population called retinal stem cells (RSCs) resided in ciliary marginal zone (CMZ). However RSCs present in all vertebrates during the retinogenesis, they just remain active in lower vertebrates (27). Nevertheless, several small cellular reservoirs of retinal stem/progenitor cells have been discovered in mammalian retina. These sources includes ciliary body epithelium (28), iris (29), limbus (30), retinal pigment epithelium (RPE) layer $(31,32)$ and muller glia region (33). Age-related macular degeneration (AMD) is one of the age-related deleterious diseases that it affects the macula which is authoritative for high-acuity daylight view in the central area of the retina. The reasons of AMD are multifactorial and contain genetic predisposition, aging, and high oxidative stress. These investigations have generated activity in micronutrients with antioxidant capabilities to inhibit the oxidative hurt elaborated in the development of degenerative eye diseases. Therefore, micronutrients including vitamins, antioxidants, and minerals are attractive as promising strategies for preventative mediation (34). Among them, retinoids and their derivatives show so fundamental function in vision that dysregulation of any genes involved in retinoid cycle in retina leads to different forms of retinal degenerations like AMD, retinitis pigmentosa (RP), and Leber congenital amaurosis (LCA). For instance, it has been recognized that any mutation in rpe65 gene cause to lack of 11-cis-retinal production in RPE cells and consequently disruption of visual cycle between photoreceptors and RPE cells, leading to progressive degeneration of rod and cone photoreceptors and RPE cells. Therefore, retinoic acid and its derivatives like retinol could help to restore the visual cycle and prevent vast cell death in retina (35).

Although, the retinal stem/progenitor cells found in mammals exhibit low capacity to generate new retinal cells, many previous studies have reported that these cells are able to proliferate and differentiate into different retinal cells under proper induction conditions in vitro (10, $36,37)$. In last years, therefore, Adult retinal stem cells 
as an ideal cell candidate received a great attentions for retinal regeneration studies. Reh and Levine (38) reported that intraocular injections of different growth factors like FGF-2 and EGF could induce RSCs derived from ciliary body epithelium to proliferate and differentiate into retinal neurons. In another study, Bertolotti et al. (39) showed that the RSCs, isolated from the adult ciliary body epithelium, could grow as neurospheres and be stimulated to differentiate into RPE cells under specific 3D culture conditions. In this regard, we isolated and cultured a stem cell population from ciliary body epithelium of mouse eyes and defined their identity as mesenchymal stem cells (MSCs). To provide more suitable conditions for MSC differentiation into retinal cells, we exploited concurrently treatment with a differentiation medium supplemented with Retinol and alginate-based hydrogels as 3D culture systems. Our results showed a reduced viability of MSCs cultured on alginate, gelatin and the mixed alg-gel scaffolds compared to $2 \mathrm{D}$ cultures, confirming that the used alginate-based scaffolds might promote MSC differentiation by preventing their self-renewal ability. Supporting the MTT data, our ICC results indicated that firstly the expression of rhodopsin protein was significantly increased in all MSCs (treated with differentiation medium with and without Retinol) cultured on alginate and alg-gel scaffolds compared to 2D cultures, indicating the promoting effects of alginate-based scaffolds on MSC differentiation. There was an exception about MSCs grown on gelatin hydrogel that showed no increased rhodopsin expression. A possible explanation for this observation is that as MSC cultures established on gelatin exhibited a low viability $(<60 \%)$ and nearly half of cell population were subjected to cell death, the physiological conditions of MSC cultures were not favorable for cell differentiation. In addition, the expression level of rhodopsin protein was significantly enhanced in Retinoltreated MSCs in comparison to those cells treated with differentiation medium without retinol. Consistent to our findings, Bi et al. (40) reported that all-trans-retinoic acid (ATRA) promoted neuronal differentiation of MSCs through activating the retinoid signaling pathway. Fawzy El-Sayed et al. (41) also showed that Retinol treatment enhanced the differentiation potential of gingival mesenchymal stem/progenitor cells (gMSCs) through activating the Wnt/ $\beta$-catenin pathway. Following the differentiation studies, our Real-Time PCR results implied on an elevated expression of Nestin (neural progenitor marker), rhodopsin (photoreceptor marker) and RPE65 (specific marker for RPE cells) genes in Retinol-treated MSCs cultured on alginate and alg-gel scaffolds, comparing to con- trols (differentiation medium without Retinol and 2D culture). The upregulation of Nestin as a neural progenitor marker in retinol-treated MSC cultures grown on alginate-based scaffolds elucidated that MSC progenies might be committed to differentiate into neural progenitors and they in turn could be considered precursors for more differentiated retinal cells like neurons and RPE cells expressing neural markers like rhodopsin and RPE65, respectively. The findings presented here confess the impressive stimulatory effects of Retinol and effectiveness of alginate-based scaffolds as an appropriate context on differentiation potential of MSC cultures.

In conclusion, the current study elucidated that Retinol treatment and the utilization of alginate-based hydrogels as $3 \mathrm{D}$ culture systems can facilitate the differentiation potential of MSCs obtained from ciliary epithelium of mouse eye into retinal progenitors and specialized cells. To light the molecular mechanisms underlying retinol function in more details, the alterations in expression levels of many more candidate genes involved in the related signaling pathways should be examined.

\section{Acknowledgments}

No acknowledgement.

\section{Potential Conflict of Interest}

The authors have no conflicting financial interest.

\section{Author Contributions}

Mahtab Haghighat carried out the experiments and wrote the MS draft; Alireza Iranbakhsh supervised the work and revised the manuscript; Javad Baharara supervised the work and validated the results; and Mostafa Ebadi and Fattah Sotoodehnejadnematalahia contributed to this work as the advisor of study designing and data analyses.

\section{References}

1. Ramachandra Rao S, Fliesler SJ. Cholesterol homeostasis in the vertebrate retina: biology and pathobiology. J Lipid Res 2021;62:100057

2. Kha CX, Guerin DJ, Tseng KA. Studying in vivo retinal progenitor cell proliferation in Xenopus laevis. Methods Mol Biol 2020;2092:19-33

3. Angileri KM, Gross JM. dnmtl function is required to maintain retinal stem cells within the ciliary marginal zone of the zebrafish eye. Sci Rep 2020;10:11293

4. Centanin L, Hoeckendorf B, Wittbrodt J. Fate restriction and multipotency in retinal stem cells. Cell Stem Cell 2011; 9:553-562 
5. Tang X, Gao J, Jia X, Zhao W, Zhang Y, Pan W, He J. Bipotent progenitors as embryonic origin of retinal stem cells. J Cell Biol 2017;216:1833-1847

6. Eymann J, Salomies L, Macrì S, Di-Poï N. Variations in the proliferative activity of the peripheral retina correlate with postnatal ocular growth in squamate reptiles. J Comp Neurol 2019;527:2356-2370

7. Jorstad NL, Wilken MS, Todd L, Finkbeiner C, Nakamura P, Radulovich N, Hooper MJ, Chitsazan A, Wilkerson BA, Rieke F, Reh TA. STAT signaling modifies Ascll chromatin binding and limits neural regeneration from muller glia in adult mouse retina. Cell Rep 2020;30:2195-2208.e5

8. Aladdad AM, Kador KE. Adult stem cells, tools for repairing the retina. Curr Ophthalmol Rep 2019;7:21-29

9. Bammidi S, Bali P, Kalra J, Anand A. Transplantation efficacy of human ciliary epithelium cells from fetal eye and Lin-ve stem cells from umbilical cord blood in the murine retinal degeneration model of laser injury. Cell Transplant 2020;29:963689720946031

10. Tropepe V, Coles BL, Chiasson BJ, Horsford DJ, Elia AJ, McInnes RR, van der Kooy D. Retinal stem cells in the adult mammalian eye. Science 2000;287:2032-2036

11. Ballios BG, Clarke L, Coles BL, Shoichet MS, Van Der Kooy D. The adult retinal stem cell is a rare cell in the ciliary epithelium whose progeny can differentiate into photoreceptors. Biol Open 2012;1:237-246

12. Canola K, Angénieux B, Tekaya M, Quiambao A, Naash MI, Munier FL, Schorderet DF, Arsenijevic Y. Retinal stem cells transplanted into models of late stages of retinitis pigmentosa preferentially adopt a glial or a retinal ganglion cell fate. Invest Ophthalmol Vis Sci 2007;48:446454

13. MacLaren RE, Pearson RA, MacNeil A, Douglas RH, Salt TE, Akimoto M, Swaroop A, Sowden JC, Ali RR. Retinal repair by transplantation of photoreceptor precursors. Nature 2006;444:203-207

14. Inoue $\mathrm{T}$, Coles BL, Dorval $\mathrm{K}$, Bremner R, Bessho $\mathrm{Y}$, Kageyama R, Hino S, Matsuoka M, Craft CM, McInnes RR, Tremblay F, Prusky GT, van der Kooy D. Maximizing functional photoreceptor differentiation from adult human retinal stem cells. Stem Cells 2010;28:489-500

15. Huang L, Chen M, Zhang W, Sun X, Liu B, Ge J. Retinoid acid and taurine promote NeuroDl-induced differentiation of induced pluripotent stem cells into retinal ganglion cells. Mol Cell Biochem 2018;438:67-76

16. Merhi-Soussi F, Angénieux B, Canola K, Kostic C, Tekaya M, Hornfeld D, Arsenijevic Y. High yield of cells committed to the photoreceptor fate from expanded mouse retinal stem cells. Stem Cells 2006;24:2060-2070

17. Macheleidt J, Mattern DJ, Fischer J, Netzker T, Weber J, Schroeckh V, Valiante V, Brakhage AA. Regulation and role of fungal secondary metabolites. Annu Rev Genet 2016; 50:371-392

18. Gudas LJ, Wagner JA. Retinoids regulate stem cell differentiation. J Cell Physiol 2011;226:322-330

19. Lee J, Cuddihy MJ, Kotov NA. Three-dimensional cell cul- ture matrices: state of the art. Tissue Eng Part B Rev 2008; 14:61-86

20. Campuzano S, Pelling AE. Scaffolds for 3D cell culture and cellular agriculture applications derived from non-animal sources. Front Sustain Food Syst 2019;3:38

21. Pan T, Song W, Cao X, Wang Y. 3D bioplotting of gelatin/alginate scaffolds for tissue engineering: influence of crosslinking degree and pore architecture on physicochemical properties. J Mater Sci Technol 2016;32:889-900

22. Farokhi M, Shariatzadeh FJ, Solouk A, Mirzadeh H. Alginate based scaffolds for cartilage tissue engineering: a review. Int J Polym Mater Polym Biomater 2020;69:230-247

23. Zarif ME. A review of chitosan-, alginate-, and gelatinbased biocomposites for bone tissue engineering. Biomater Tissue Eng Bull 2018;5:97-109

24. Geiger P, Barben M, Grimm C, Samardzija M. Blue lightinduced retinal lesions, intraretinal vascular leakage and edema formation in the all-cone mouse retina. Cell Death Dis 2015;6:e1985

25. Carr AJ, Vugler AA, Hikita ST, Lawrence JM, Gias C, Chen LL, Buchholz DE, Ahmado A, Semo M, Smart MJ, Hasan S, da Cruz L, Johnson LV, Clegg DO, Coffey PJ. Protective effects of human iPS-derived retinal pigment epithelium cell transplantation in the retinal dystrophic rat. PLoS One 2009;4:e8152

26. Haghighat $M$, Iranbakhsh $A$, Baharara $J$, Ebadi $M$, Sotoodehnejadnematalahi F. Effect of $\beta$-carotene on the differentiation potential of ciliary epithelium-derived MSCs isolated from mouse eyes on alginate-based scaffolds. Exp Eye Res 2021;202:108346

27. Yu JJ, Azzam DB, Chwa M, Schneider K, Cho JH, Hsiang C, Klassen H, Kenney MC, Yang J. Age-related macular degeneration (AMD) transmitochondrial cybrids protected from cellular damage and death by human retinal progenitor cells (hRPCs). Stem Cells Int 2021;2021:6655372

28. Del Debbio CB, Peng X, Xiong H, Ahmad I. Adult ciliary epithelial stem cells generate functional neurons and differentiate into both early and late born retinal neurons under non-cell autonomous influences. BMC Neurosci 2013;14: 130

29. Yamamoto N, Hiramatsu N, Ohkuma M, Hatsusaka N, Takeda S, Nagai N, Miyachi EI, Kondo M, Imaizumi K, Horiguchi M, Kubo E, Sasaki H. Novel technique for retinal nerve cell regeneration with electrophysiological functions using human iris-derived iPS cells. Cells 2021;10:743

30. Notara M, Lentzsch A, Coroneo M, Cursiefen C. The role of limbal epithelial stem cells in regulating corneal (Lymph)angiogenic privilege and the micromilieu of the limbal niche following UV exposure. Stem Cells Int 2018; 2018:8620172

31. Akrami H, Soheili ZS, Khalooghi K, Ahmadieh H, Rezaie-Kanavi M, Samiei S, Davari M, Ghaderi S, Sanie-Jahromi F. Retinal pigment epithelium culture;a potential source of retinal stem cells. J Ophthalmic Vis Res 2009;4:134-141

32. Fischer AJ, Reh TA. Transdifferentiation of pigmented epi- 
thelial cells: a source of retinal stem cells? Dev Neurosci 2001;23:268-276

33. Langhe R, Pearson RA. Rebuilding the retina: prospects for Müller glial-mediated self-repair. Curr Eye Res 2020;45: 349-360

34. Khoo HE, Ng HS, Yap WS, Goh HJH, Yim HS. Nutrients for prevention of macular degeneration and eye-related diseases. Antioxidants (Basel) 2019;8:85

35. Palczewski K. Retinoids for treatment of retinal diseases. Trends Pharmacol Sci 2010;31:284-295

36. Coles BL, Angénieux B, Inoue T, Del Rio-Tsonis K, Spence JR, McInnes RR, Arsenijevic Y, van der Kooy D. Facile isolation and the characterization of human retinal stem cells. Proc Natl Acad Sci U S A 2004;101:15772-15777

37. Xu H, Sta Iglesia DD, Kielczewski JL, Valenta DF, Pease ME, Zack DJ, Quigley HA. Characteristics of progenitor cells derived from adult ciliary body in mouse, rat, and human eyes. Invest Ophthalmol Vis Sci 2007;48:1674-1682

38. Reh TA, Levine EM. Multipotential stem cells and progenitors in the vertebrate retina. J Neurobiol 1998;36:206-220

39. Bertolotti E, Neri A, Camparini M, Macaluso C, Marigo V. Stem cells as source for retinal pigment epithelium transplantation. Prog Retin Eye Res 2014;42:130-144

40. Bi Y, Gong $\mathrm{M}$, Zhang X, Zhang X, Jiang W, Zhang Y, Chen J, Liu Y, He TC, Li T. Pre-activation of retinoid signaling facilitates neuronal differentiation of mesenchymal stem cells. Dev Growth Differ 2010;52:419-431

41. Fawzy El-Sayed KM, Hein D, Dörfer CE. Retinol/inflammation affect stemness and differentiation potential of gingival stem/progenitor cells via Wnt/ $\beta$-catenin. J Periodontal Res 2019;54:413-423 